\title{
Effect of Low-Dose Irradiation on the Surface Properties of Poly[(trifluoroethoxy)(telomer fluoro alkoxy)phosphazene]
}

\author{
W. M. REICHERT ${ }^{*}$ F. E. FILISKO, $\dagger$ AND S. A. BARENBERG $\ddagger$ \\ *Department of Bioengineering, University of Utah, Salt Lake City, Utah; †Department of Materials Science, \\ University of Michigan, Ann Arbor, Michigan 48109; and $\ddagger$ E.I. Du Pont de Nemours \& Co., \\ Experimental Station 323, Wilmington, Delaware 19898
}

Received August 23, 1983; accepted April 30, 1984

\begin{abstract}
The effect of low-dose ultraviolet and $\gamma$ irradiation on the surface and bulk properties of poly[(trifluoroethoxy)(telomer fluoro alkoxy)phosphazene], was found to initially result in an increased side chain mobility followed by a decreased mobility. The initial increase is attributed to partial cleavage of selected side chains followed by a decreased mobility as a result of interside chain crosslinking. These above effects and the solid state characterization of the polymers were documented by dynamic mechanical analysis, difference dielectric spectroscopy, electron microscopy, electron spectroscopy for chemical analysis, attenuated total internal reflection spectroscopy, contact angle measurements, differential scanning calorimetry, gel permeation chromatography, tensile stress strain, and mass spectroscopy.
\end{abstract}

\section{INTRODUCTION}

The effect of low-dose ultraviolet and $\gamma$ irradiation on the surface and bulk properties of poly[(trifluoroethoxy)(telomer fluoro alkoxy)phosphazene], PNF, were documented by dynamic mechanical analysis, dielectric spectroscopy, tensile stress-strain, electron microscopy, X-ray dispersion analysis, electron spectroscopy for chemical analysis, attenuated total internal reflection infrared spectroscopy, contact angle, differential scanning calorimetry, gel permeation chromatography, and mass spectroscopy.

The dynamic mechanical work of Connelly and Gillham (1) on poly[bis(trifluoroethoxy)phosphazene], PTF (Fig. 1b), and poly[(trifluoroethoxy)(telomer fluoroalkoxy)phosphazene], PNF (Fig. 1a) revealed an $\alpha$ relaxation for both polymers occurring at $-50^{\circ} \mathrm{C}$, with the PNF exhibiting a $\beta$ relaxation at $-140^{\circ} \mathrm{C}$. Connelly and Gillham (1) ascribed the $\alpha$ relaxation to the glass transition and the $\beta$ relaxation to the onset of the fluoroalkoxy side chain motion. They did not observe the $\beta$ relaxation for the trifluoriethoxy side chains.

O'Brien (2) and Hiraoka (3) studied the effect of high-dose ultraviolet irradiation on poly[phosphazenes] with respect to the utilization of these molecules as photoresistant materials and to the mechanism of photolysis. In these studies $(2,3)$ and others (4-6) little mention is made as to the effect of crosslinking, changes in the primary and secondary molecular motions, and surface and/or bulk chemistry, as a function of irradiation except that the exposed polymers became less soluble.

It is therefore the intent of this paper to present further evidence indicating the effects of low-dose irradiation on the physical properties of poly[(fluoroalkoxy)phosphazenes].

\section{MATERIALS AND METHODS}

\section{Polymers}

The poly[bis(trifluoroethoxy)phosphazene], PTF (Fig. 1b), and poly[(trifluoroethoxy)- 


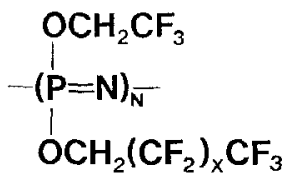

(a)

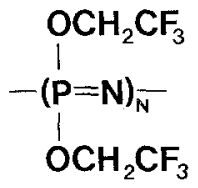

(b)
FIG. 1. (a) Poly[(trifluoroethoxy)(telomer fluoroalkoxy)phosphazene], PNF. (b) Poly[bis(trifluoroethoxy)phosphazene], PTF.

(telomer fluoroalkoxy)phosphazene], PNF (Fig. 1a, used were obtained through the courtesy of Firestone Research (7). PTF, which is disubstituted with trifluoroethoxy side chains, is the only poly[(fluoroalkoxy)phosphazene] extensively studied by dielectric and dynamic mechanical methods. We used PTF as a documented standard to which the thermomechanical and dielectric PNF results were compared thus helping us to differentiate between the mobilities of the trifluoroethoxy and telomer fluoroalkoxy side chains of PNF.

\section{Polymer Purification and Film Casting}

The polymers were dissolved in Freon TA (Miller-Stephenson) followed by mixing in an equal volume of distilled, deionized water with the Freon-polymer solution. The system was allowed to phase-separate. The Freon layer was then eluted into hexane causing the high-molecular-weight polymer to be precipitated out. Solutions of the purified polymer $(10 \% \mathrm{w} / \mathrm{v})$ were prepared in acetone. The films used in the dynamic mechanical, contact angle and ESCA studies were cast from these solutions in a closed environment to retard the rate of solvent evaporation and reduce surface contamination. This method produced smooth isotropic films with a thickness of $0.015 \mathrm{~cm}$. The prepared films were vacuum-dried for $12 \mathrm{hr}$ to remove the residual solvent.

\section{Ultraviolet Irradiation}

The UV irradiation of the polymer was done with a Hanovia 616A high-pressure quartz mercury vapor lamp with an output in the UV range of $70 \mathrm{~W} / \mathrm{cm}^{2}$ at a distance of $50 \mathrm{~cm}$. Unless stated otherwise, the samples used in each experiment were irradiated for 0,1 , and $24 \mathrm{hr}$ in air at ambient temperatures.

\section{Dielectric Measurements}

Dielectric measurements were performed with an automated dielectric system (8). Thin films of the purified polymer (on the order of $1 \mathrm{~mm}$ ) were cast at room temperature directly onto the cell from three successive $5-\mathrm{ml}$ applications of $1.0 \%(\mathrm{w} / \mathrm{v})$ acetone solution. Each application was allowed to dry in a closed environment. After a suitable film was obtained, it was vacuum-dried for $12 \mathrm{hr}$ at $100^{\circ} \mathrm{C}$. The original films were run, irradiated for $1 \mathrm{hr}$, rerun, irradiated for 23 more hours, and rerun again allowing us to observe the effects of irradiation on the same sample. The temperature range investigated was from -200 to $200^{\circ} \mathrm{C}$, over a frequency range of $0.234-20.0 \mathrm{KHz}$.

Difference dielectric measurements were obtained by placing the unirradiated polymer spectra in the memory of the dielectric system, and subtracting the unirradiated spectra point by point from the irradiated spectra.

\section{Dynamic Mechanical Measurements}

A Toyo Rheovibron DDV-11 was used for dynamic mechanical analysis of polymer samples cut from acetone cast films. The samples $(0.5 \times 4.0 \times 0.015 \mathrm{~cm})$ were UVirradiated prior to the measurements. The test chamber was cooled to approximately $-175^{\circ} \mathrm{C}$ and readings were taken at $110 \mathrm{~Hz}$ as the sample temperature ramped $\left(1^{\circ} \mathrm{C} /\right.$ min) to $-70^{\circ} \mathrm{C}$ at which point it was not possible to obtain the critical tension required for Rheovibron measurements.

\section{Contact Angle}

The advancing contact angle and critical surface tension was measured in conjunction with Baier (9). All measurements were performed at $20^{\circ} \mathrm{C}$. 


\section{ESCA}

The ESCA measurements were performed by Ratner (10). The instrument used was a HP 5950B ESCA system employing an 800$\mathrm{W}$ monochromatized X-ray beam from an aluminum anode. All measurements were done at ambient temperatures.

\section{TEM and EDAX}

TEM and EDAX were done in a JEOL $100 \mathrm{C}$ equipped with a Princeton Gamma Tech detector. Thin films of the polymer were cast from acetone $(0.1 \% \mathrm{w} / \mathrm{v})$ onto carbon-coated glass slides. The films were placed in contact with isotonic saline for 4 $\mathrm{hr}$ and then washed in deionized distilled water. The polymer films were then lifted from the slides onto copper grids. All photomicrographs and dispersion maps were taken at $100,000 \times$.

\section{Bound Water}

The amount of bound water associated with PNF was measured using a PerkinElmer DSC II equipped with a subambient stage. The samples were scanned from 227 to $303^{\circ} \mathrm{K}$ at $10^{\circ} \mathrm{K} / \mathrm{min}$ under dry nitrogen. The heats of fusion of the sorbed water were calculated relative to the heats of fusion of indium (11).

\section{$G P C$}

Solutions $0.1 \%(\mathrm{w} / \mathrm{v})$ of irradiated and unirradiated polymer were made in a $0.1 \%$ $(\mathrm{v} / \mathrm{v}) \mathrm{THF}$ in acetone solvent. The solutions were filtered with $1-\mu \mathrm{m}$ Waters filters prior to injection into the GPC to remove any insoluble gel. The instrument used was a Waters liquid chromatograph equipped with two microstyragel columns in series with porosities of 105 and $106 \AA$. The flow rate was $2.0 \mathrm{ml} / \mathrm{min}$ at ambient temperatures.

\section{Mass Spectroscopy}

A small spigoted Pyrex vessel provided a closed chamber in which 0.2 -g polymer sam- ples were placed and irradiate under vacuum thus trapping the volatile decomposition products. The photolytic by-products were analyzed by direct injection of the volatiles into a Finnigan 4000 mass spectrometer. In this study $\mathrm{Co}^{60} \gamma$ irradiation doses of 0.1 , 1.0, and 10.0 Mrad were used because UV light could not penetrate the Pyrex and impinge upon the sample to produce a sufficient level of detectable photodegradation byproducts.

\section{Tensile Stress-Strain}

Stress-strain measurements of PNF were performed on an Instron tensile tester. The sample dimensions were $0.5 \times 2.0 \times 0.015$ $\mathrm{cm}$. All measurements were done at ambient temperatures with a strain rate of 0.2 in $/ \mathrm{min}$.

\section{FTIR (IR/ATIR)}

The FTIR samples were cast onto KRS-5 thallium-bromide-iodide crystals from a $0.1 \%(\mathrm{w} / \mathrm{v})$ solution of purified PNF in acetone. Covering the crystal surface with a thin coating of the solution produced a polymer film of less than $1 \mu \mathrm{m}$ in thickness. All films were cast in a closed environment. A Digilab FTS-10 interferometer at Battelle Labs (12) was used for attenuated total internal reflectance infrared analysis (ATIR), and Digilab FTS-200 Interferometer was used for conventional transmission infrared spectroscopy (IR). Both systems provided a resolution of $4 \mathrm{~cm}^{-1}$. The number of scans per sample was 200 for the ATIR and 1000 for the IR.

TABLE I

Critical Surface Tension of PNF as a Function of UV Irradiation

\begin{tabular}{cc}
\hline $\begin{array}{c}\text { UV irradiation } \\
\text { (hr) }\end{array}$ & $\begin{array}{c}\text { Critical surface tension } \\
\text { (dyn } / \mathrm{cm})\end{array}$ \\
\hline 0 & 16.5 \\
1 & 14.4 \\
24 & 15.6 \\
\hline
\end{tabular}

Journal of Colloid and Interface Science, Vol. 101, No. 2, October 1984 


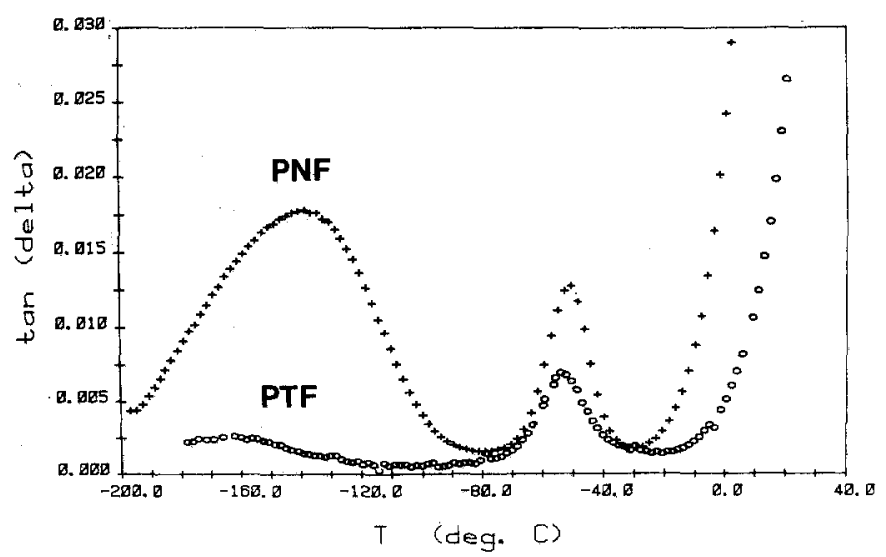

FIG, 2. Two dielectric transitions exhibited by PNF $(+)$ and PTF $(0)$ taken at $0.234 \mathrm{kHz}$.

\section{RESULTS}

The morphology of unirradiated PNF (8, 13 ), as cast from acetone, exhibited a homogeneous morphology with no apparent indication of any order: The X-ray dispersion map of the unirradiated polymer exhibited a homogeneous distribution of phosphorous with no evidence of aggregation $(3,13,14)$. The morphology of the 1- and 24-hr irradiated PNF's, as those above, also exhibited homogeneous morphologies (8). The X-ray dispersion maps of irradiated PNF's indicated no change in the phosphorous distribution.

The result of the contact angle measurements (Table I) of irradiated and unirradiated PNF's indicate there is no change in the surface chemistry of PNF, per se. This study also showed that the PNF surface is comprised almost entirely on the fluorinated side chains $(8,13)$.

The dielectric measurements (Fig. 2) of the as cast PNF and PTF polymers exhibited three relaxations. The lower temperature $\beta$ relaxations of PNF and PTF $(-140$ and $-164^{\circ} \mathrm{C}$, respectively) are ascribed to the onset of side chain motion independent of the backbone. The $\alpha$ relaxation $\left(-55^{\circ} \mathrm{C}\right)$ is associated with the initiation of segmental backbone motion. These assignments are in agreement with the work of Connelly and Gillhan (1). The origin of the third transition $\left(0^{\circ} \mathrm{C}\right)$ is questionable, but for reasons discussed later, it appears that this large shoulder could be associated with bound water.

In PTF the $\beta$ relaxation results from the

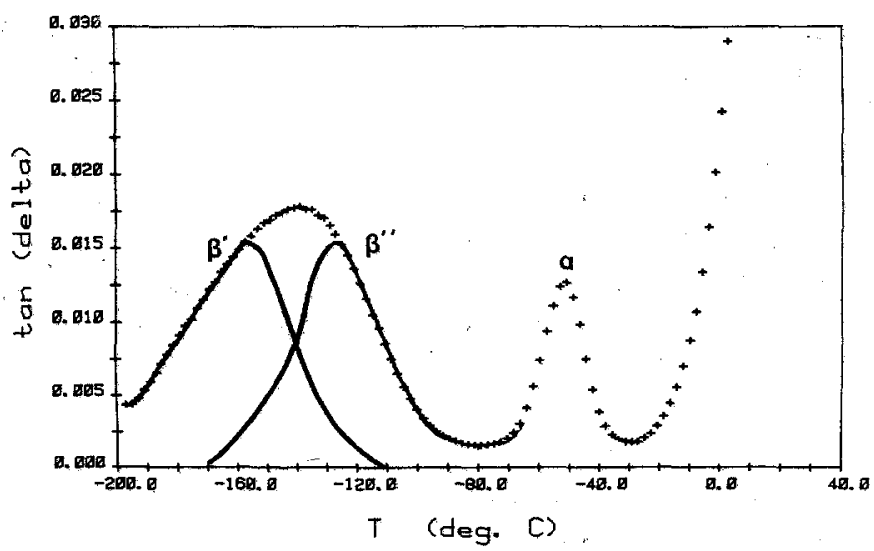

FIG. 3. Deconvolution of $\beta$ transition of PNF into $\beta^{\prime}$ and $\beta^{\prime \prime}$. 


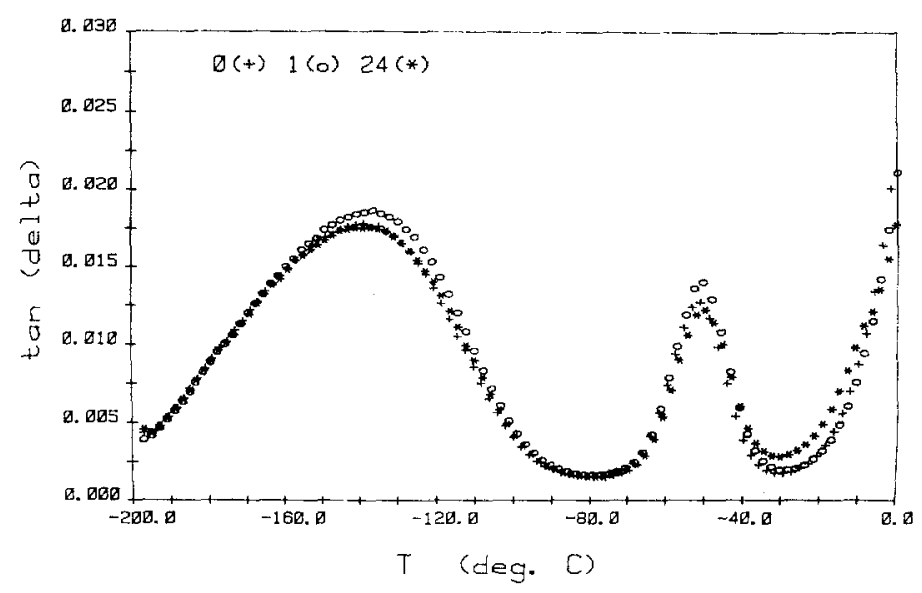

FIG. 4. Dielectric transitions of PNF as a function of ultraviolet irradiation.

motion of the trifluoroethoxy side chains (Fig. 2), however, in the PNF this peak represents the combined relaxations of the trifluoroethoxy and the larger telomer fluoroalkoxy side chains (Fig. 2). From an activation energy argument the longer the side chain the higher the temperature required for the onset of mobility. Based on different lengths of the side chains, it was reasonable to assume that the $\beta$ relaxation of PNF can be resolved into two portions corresponding to the longer and shorter side chains. Analysis of this type would then infer that the lowtemperature end of the $\beta$ relaxation of PNF could arise from the onset of trifluoroethoxy motion $\left(-160^{\circ} \mathrm{C}\right)$, with the upper temperature portion $\left(-140\right.$ to $\left.-120^{\circ} \mathrm{C}\right)$, being ascribed primarily to the telomeric fluoroalkoxy side groups. In Fig. 3 we show a schematic representation of how two peaks may produce a broad one.

When PNF was irradiated with ultraviolet light for 1 and $24 \mathrm{hr}$, small but significant changes could be observed in the $\alpha$ and $\beta$ relaxations (Fig. 4). Specifically, the magnitudes of these relaxations increased after 1 $\mathrm{hr}$ of ultraviolet irradiation, then decreased after $24 \mathrm{hr}$ of exposure.

In order to take a closer look at the effect of ultraviolet irradiation on PNF a difference

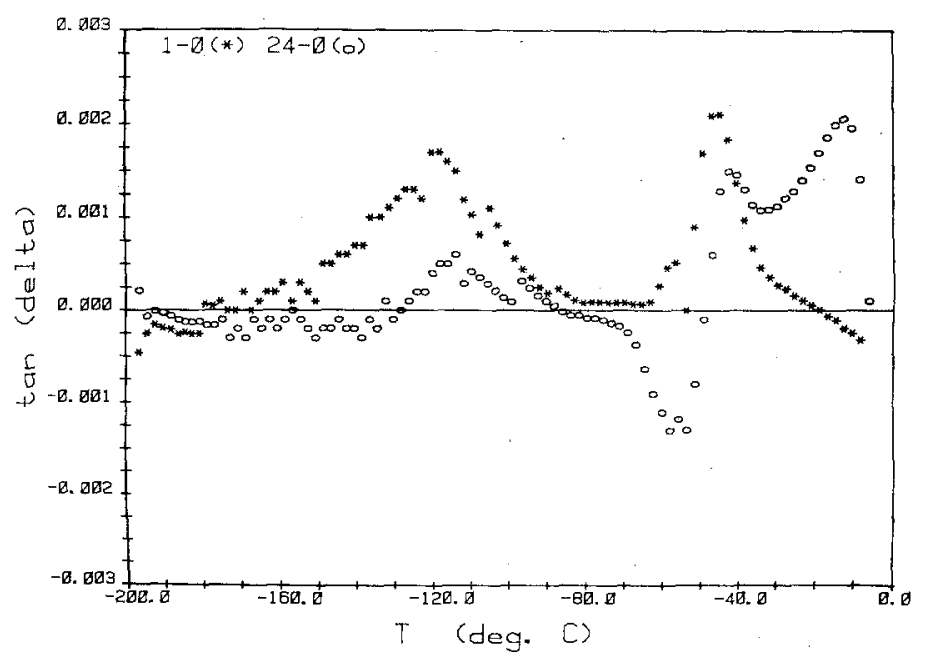

FIG. 5. Difference dielectric spectral of data shown in Fig. 4. 


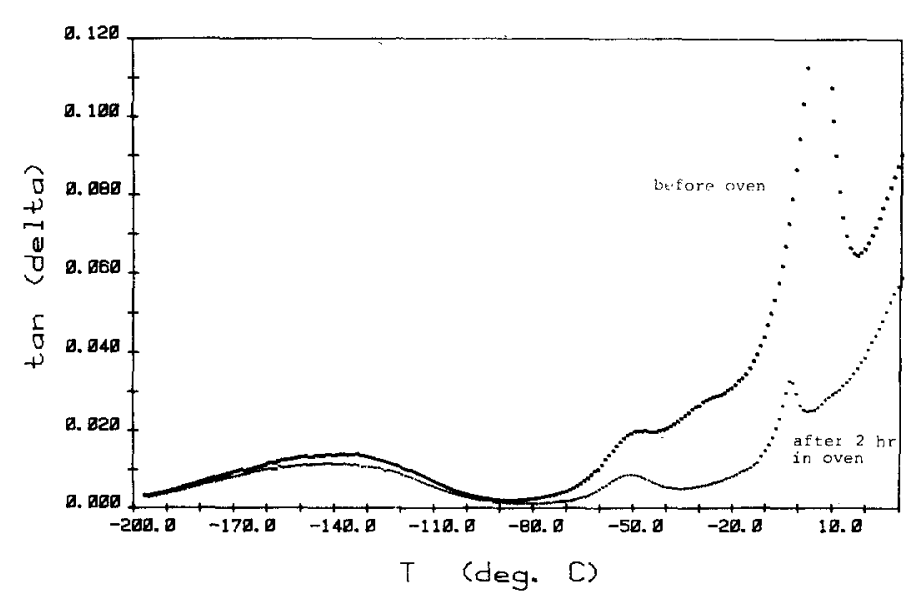

FIG. 6. Dielectric water spike at $0^{\circ} \mathrm{C}$ before and after vacuum drying.

dielectric method was developed (8). With this technique small differences between the dielectric spectra become quite discernable, emphasizing any changes in peak shape or location. Figure 5 shows the two different spectra (1-0 and 25-0 hr subtractions) derived from the data shown in Fig. 4. Some intermediate irradiation times were also tested (1/ $4-0,1 / 2-0,6-0)$ which showed that the actual $\alpha$ and $\beta$ peak maxima occurred after $1 / 2 \mathrm{hr}$ of irradiation followed by a continual decrease with further irradiation (8).

The skewed changes in the $\beta$ relaxation of PNF were of particular interest (Fig. 5). The observed increase and subsequent decrease occurred almost exclusively between -140 and $-100^{\circ} \mathrm{C}$ with little to no change in the $-160^{\circ} \mathrm{C}$ portion of the $\beta$ relaxation. These data suggest, in conjunction with the deconvolution discussion, that the telomeric fluoroalkoxy pendent groups are involved in a photoinduced change in mobility while the trifluoroethoxy side chains remain relatively unaffected. Additionally, the difference spectra revealed the $\alpha$ transition was shifted to a higher temperature after $24 \mathrm{hr}$ of irradiation.

Due to the proximity of the high-temperature transition to $0^{\circ} \mathrm{C}$ (Fig. 2) it is a natural assumption that water is involved. When a PNF film is submerged in water followed by air-drying a large dielectric spike at $0^{\circ} \mathrm{C}$ is superposed upon the observed shoulder (Fig.
6). If the sample is vacuum-dried at $100^{\circ}$ the spike and shoulder continue to decrease until the spike eventually disappears after $12 \mathrm{hr}$ of drying. However, a residual shoulder remains in spite of continued drying efforts up to $24 \mathrm{hr}$.

The disappearance of this water spike does not imply that all of the water has been removed from the sample. The water associated with polymers can be divided into bulk (or freezable) water, and bound water. This distinction implies that elimination of the water spike at $0^{\circ} \mathrm{C}$ only signals the sample is free of bulk water while the residual shoulder is perhaps associated with the individual water molecules which remain bound to the molecule. However, there exists no direct evidence to support this conclusion.

Differential scanning calorimetry was performed in order to ascertain the degree of bound water associated with the PNF polymer

\section{TABLE II}

Bound Water Content of PNF as a Function of UV Irradiation

\begin{tabular}{cc}
$\begin{array}{c}\text { UV irradiation } \\
(\mathrm{hr})\end{array}$ & $\begin{array}{c}\text { mg Bound water/ } \\
\text { mg polymer }\end{array}$ \\
\hline 0 & 0.01 \\
1 & 0.01 \\
24 & 0.05 \\
\hline
\end{tabular}




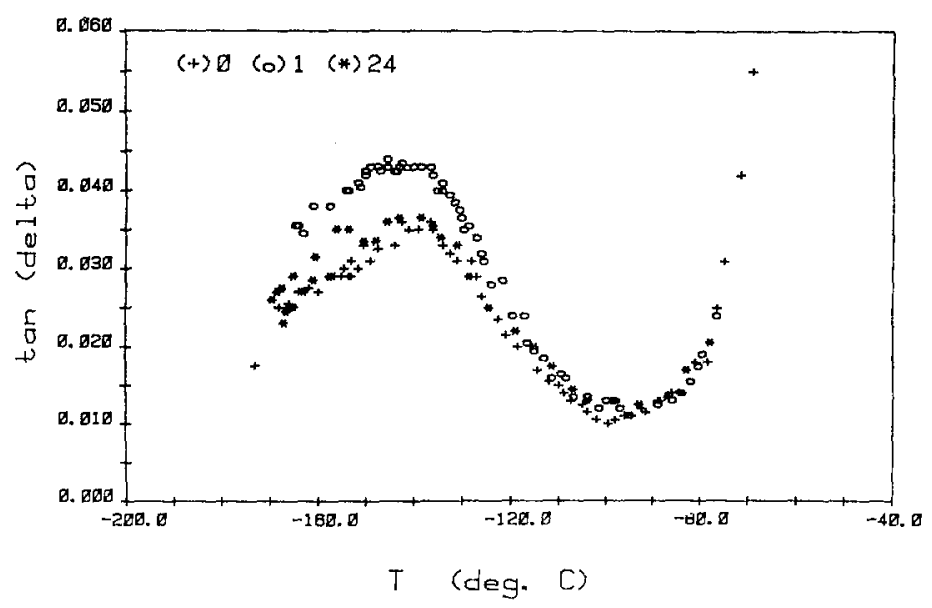

FIG. 7. Thermomechanical transitions of PNF as a function of ultraviolet irradiation taken at $110 \mathrm{kHz}$.

(8). As can be observed in Table II the irradiated PNF contained $0.01 \mathrm{mg}$ bound water/mg polymer which increased to a maximum of $0.05 \mathrm{mg}$ bound water $/ \mathrm{mg}$ polymer.

Another important aspect of this study is the effect of bound water content on the dielectric relaxation of PNF. Of the polymers studied to date, it was found that enhancement of their low-temperature relaxation peaks often occurred in proportion to the samples of bound-water content. If this effect was taking place in PNF one would expect, based on the dielectric results, that the 1-hr irradiated sample would bind the largest amount of water with the 0 - and 24-hr samples binding the least. This expectation is not substantiated by Table II. These data indicate that the $\beta$ relaxation changes observed in Figs. 4 and 5 are the result of differences inside chain mobility.

Dielectric spectra were also taken of PNF samples irradiated in a nitrogen blanket (8). Under these conditions the relaxation peak continued to increase with increased time of ultraviolet exposure. These results indicate a significant role of oxygen in the $\beta$ relaxation decrease after $24 \mathrm{hr}$ of irradiation.

Dynamic mechanical measurements were undertaken to confirm the dielectric studies. This study was limited in that the viscoelastic properties of the PNF would not permit the taking of Rheovibron measurements beyond the glass transition temperature. As a result, only the $\beta$ relaxation and the onset of the $\alpha$ relaxation was documented. In spite of this short coming, the dynamic mechanical results (Fig. 7) exhibited a $\beta$ relaxation enhancement $(1 \mathrm{hr})$ followed by a decrease $(24 \mathrm{hr})$. This trend is identical to that observed in the dielectric results (Fig. 4).

The dielectric and thermomechanical relaxation changes observed in these two studies indicate the mobility of the side chains ultimately become restricted as evidenced by the relaxation peak decrease after $24 \mathrm{hr}$ of irradiation. This restriction in motion is further substantiated by (1) the upward shift in the $\alpha$ transition after $24 \mathrm{hr}$ of irradiation, and (2) the fact that the 0 - and 1-hr irradiated PNF samples dissolved in acetone, whereas the 24-hr irradiated samples swelled. ${ }^{1}$ All of these observations support the production of a crosslinked network. Stemming from these results additional studies were undertaken to further elucidate the effect of ultraviolet irradiation on PNF.

The ESCA results (Table III) of irradiated and unirradiated PNF $(8,10)$ show the nitrogen-carbon and phosphorous-carbon ratios remaining relatively constant in the $0-, 1-$, and 24-hr irradiated samples. However, the

\footnotetext{
${ }^{1}$ Samples irradiated up to $24 \mathrm{hr}$ under nitrogen did not swell in acetone.
} 
TABLE III

Normalized ESCA Peak Intensity Ratios

\begin{tabular}{ccccc}
\hline $\begin{array}{c}\text { UV iradiation } \\
\text { (hr) }\end{array}$ & P/C & N/C & O/C & F/C \\
\hline 0 & 0.20 & 0.16 & 0.33 & 1.47 \\
1 & 0.22 & 0.17 & 0.32 & 1.57 \\
24 & 0.22 & 0.17 & 0.35 & 1.64 \\
\hline
\end{tabular}

fluorine-carbon ratio increased continuously $(0>1>24)$. Considering the chemical structure of PNF, these results suggest the photoinduced reaction occurs within the side chains with little involvement of the backbone. There also appears to be a slight increase in the oxidation level of PNF with irradiation which could explain the small increase in bound water in the 24-hr irradiated PNF. Furthermore, the ESCA studies indicated a decrease in carbon peaks associated with the fluorinated chains accompanied by the appearance of an aliphatic peak after $24 \mathrm{hr}$ of irradiation (Table IV). These results (Tables III and IV) could result from side chain cleavage which in turn serves as a potential source of radical formation and hence crosslinking.

Subtraction spectra from the ATIR study $(8,12,13)$ also indicated a reduction in bands that can be attributed to the elimination of fluorocarbon groups in the 24-hr irradiated samples $(1250,1170$, and 1090 $\mathrm{cm}^{-1}$ ). There was also a decrease in the $=\mathrm{C}-\mathrm{H}$ olefinic band $\left(965 \mathrm{~cm}^{-1}\right)$ which suggests the destruction of the unsaturated side chains added to PNF as cure sites (8). Like the ESCA results, the ATIR samples irradiated for $1 \mathrm{hr}$ showed little change and did not be evident until after $24 \mathrm{hr}$ of exposure.

The volatiles evolved from irradiated PNF samples as measured by mass spectroscopy (Table V) were analyzed to be the photolytic by-products of side chain cleavage (8). $\mathrm{Co}^{60}$ $\gamma$ irradiation was used to ensure sufficient irradiative degradation of the PNF and thus the production of volatile by-products at detectable levels. The results of Table $\mathrm{V}$ support the primary photosusceptibility of
TABLE IV

Percent Area Contribution of $\mathrm{C}_{1 s}$ Peaks to Total Carbon Signal

\begin{tabular}{rrrrc}
\hline $\begin{array}{c}\text { UV irradiation } \\
(\mathrm{hr})\end{array}$ & $\begin{array}{c}-\mathrm{CF}_{3}- \\
(293.2 \mathrm{eV})\end{array}$ & $\begin{array}{c}-\mathrm{CF}_{2}- \\
(291.4 \mathrm{eV})\end{array}$ & $\begin{array}{c}-\mathrm{OCH}_{2}- \\
(288.0 \mathrm{eV})\end{array}$ & $\begin{array}{r}-\mathrm{CH}^{2}- \\
(285.0 \mathrm{eV})\end{array}$ \\
\hline 0 & $28 \%$ & $36 \%$ & $36 \%$ & - \\
1 & $28 \%$ & $36 \%$ & $36 \%$ & - \\
24 & $26 \%$ & $36 \%$ & $34 \%$ & $3 \%$ \\
\hline
\end{tabular}

the side chains and relative stability of the backbone.

Finally, the existence of a crosslinked network was confirmed by tensile stress-strain analysis of ultraviolet-irradiated PNF samples (8). This analysis showed the transformation of PNF from a soft-weak viscoelastomer (0 hr of UV) to a lightly crosslinked rubber ( 24 hr of UV). In addition, the involvement of the backbone in the photolysis/crosslinking reaction was discounted by GPC studies which showed no significant increase in the polydispersity index of the soluble fraction of irradiated PNF (8).

\section{DISCUSSION}

The results of the dynamic mechanical and dielectric studies of PNF as a function of ultraviolet exposure both showed a $\beta$ relaxation enhancement after $1 \mathrm{hr}$ of irradiation followed by a decrease after $24 \mathrm{hr}$ of irradiation. The fact that the two techniques produced the same results at the same temperatures lead to the following conclusion:

\section{TABLE V}

Volatile By-Products from $\mathrm{Co}^{60} \gamma$ Irradiated PNF

\begin{tabular}{|c|c|c|c|}
\hline \multirow{2}{*}{$\begin{array}{c}\text { Mass } \\
(\mathrm{g} / \mathrm{mole})\end{array}$} & \multirow{2}{*}{$\begin{array}{l}\text { Assigned } \\
\text { structure }\end{array}$} & \multicolumn{2}{|c|}{$\begin{array}{l}\text { Estimated relative abundance } \\
\text { above background }\end{array}$} \\
\hline & & $1.0 \mathrm{Mrad}$ & $10.0 \mathrm{Mrad}$ \\
\hline $44,45,48$ & $\mathrm{CO}_{2}$ & 18 & 14 \\
\hline 51 & $\mathrm{CF}_{2} \mathrm{H}^{+}$ & 1 & 2 \\
\hline 61 & $\mathrm{CF}_{2} \mathrm{CH}_{2}^{+}$ & & 1 \\
\hline 69 & $\mathrm{CF}_{3}^{+}$ & & 1 \\
\hline 81 & $\mathrm{CF}_{2} \mathrm{~F}_{3}^{+}$ & & 3 \\
\hline
\end{tabular}


the identical molecular phenomenon was being observed. Specifically, we had observed an increase in side chain motion in the $1-\mathrm{hr}$ irradiated samples followed by a decrease in this motion in samples irradiated for $24 \mathrm{hr}$.

The ESCA, mass spectroscopy, and ATIR results suggest a slight photolytic cleavage of the pendent side chains of PNF with irradiation that evidently did not reach detectable levels until after $24 \mathrm{hr}$ of irradiation. Based on these data, we contend the increased motion which leads to the $\beta$ relaxation enhancement results from the partial cleavage of some of the side chains. As a result, side chains at or near the point of cleavage would experience an increased freedom of motion in the 1-hr irradiated samples.

The tensile stress-strain (8) and solubility results support the formation of a crosslinked network in the samples irradiated for $24 \mathrm{hr}$ in air. Additionally, the ESCA carbon spectra showed the formation new carbon-carbon aliphatic bonds solely in the 24-hr irradiated sample which has been attributed to the formation of interside chain crosslinks in PTF (3). These data lead toward the contention that the major factor contributing to the relaxation decrease is the formation of enough interside chain crosslinks to significantly restrict side chain motion.

The actual mechanism of crosslinking is not entirely obvious from the above data, but the results of these studies in conjunction with parallel experiments performed in a nitrogen environment (8) suggest a major role played by oxygen. This conclusion is primarily supported by the fact that samples irradiated up to $24 \mathrm{hr}$ under nitrogen did not swell in acetone. The following are some plausible crosslinking reactions which can occur at the site of side chain photolysis and in turn restrict the side chain mobility of PNF (8).
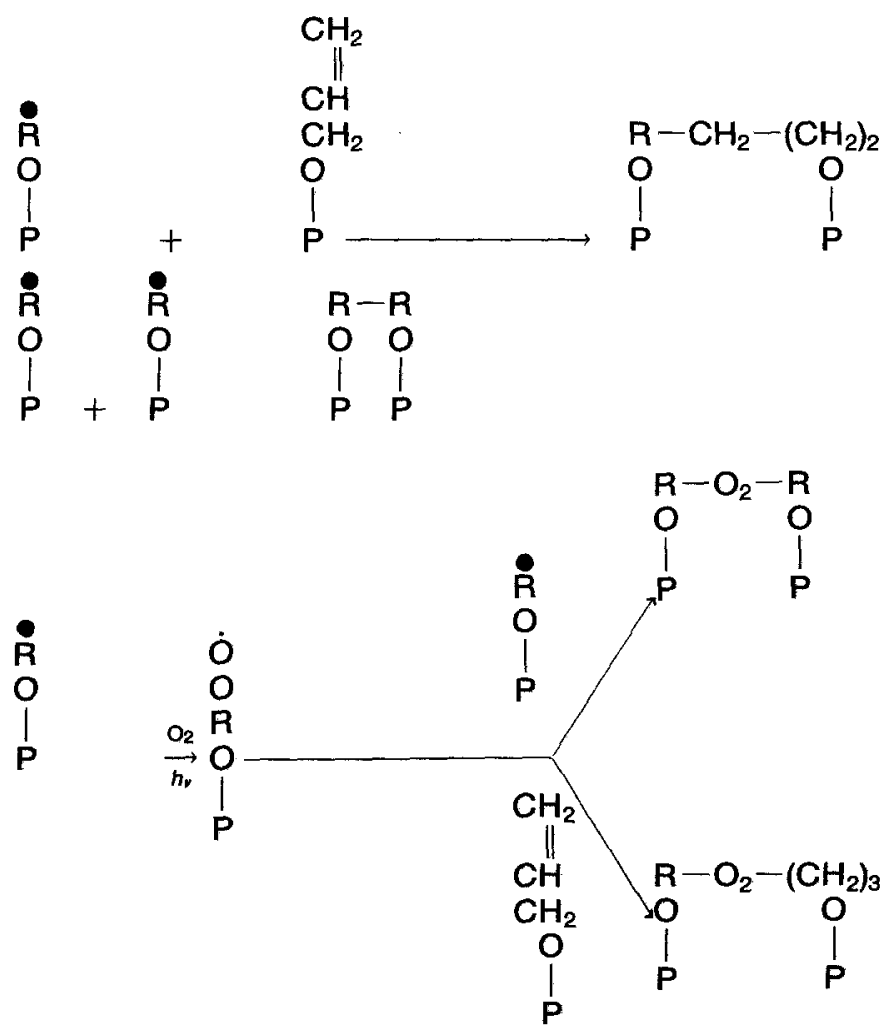

$\left(\mathrm{R}=\mathrm{CH}_{2}, \mathrm{CH}_{2} \mathrm{CF}_{2}, \mathrm{CH}_{2}\left(\mathrm{CF}_{2}\right)_{x}\right.$, where $\left.x=3,5,7\right)$ 


\section{REFERENCES}

1. Connelly, T. M., and Gillham, J. K., J. Appl. Polym Sci. 20, 473 (1976).

2. O'Brien, J. P., Ferrar, W. T., and Allcock, H. R., Macromolecules 12(1), 108 (1979).

3. Hiraoka, H., Lee, W., Welsch, L. W., and Allen, R. W., Macromolecules 12(4), 753 (1979).

4. Beggiato, G., Bordin, P., Minto, F., and Busulini, L., London Polym. J. 15, 403 (1979).

5. Bortolus, P., Minto, F., Beggiato, G., and Lora, S., J. Appl. Polym. Sci. 24, 285 (1979).

6. Gleria, M., Minto, F., Lora, S., and Bortolus, P., Eur. Polym. J. 15, 671 (1979).

7. Firestone Central Research, 1200 Firestone Parkway, Akron, Ohio.

8. Reichert, W. M., PhD. Dissertation, "Poly(organo- phosphazenes): Characterization and Hemocompatibility," University of Michigan, Ann Arbor, Mich., 1981.

9. Baier, R. E., Calspan Corp., P.O. Box 440, Buffalo, N. Y.

10. Ratner, B. D., Department of Chemical Engineering, University of Washington, Seattle, Wash.

11. Garcia, C., Anderson, J. M., and Barenberg, S. A., Trans. Amer. Soc. Artif. Int. Organs 26, 294 (1980).

12. Gendreau, M., Battelle, 505 King Ave., Columbus, Ohio.

13. Reichert, W. M., Filisko, F. E., and Barenberg, S. A., "Biomaterials: Interfacial Phenomenon and Applications." Adv. Chem. Sciences, Vol. 199, p. 177.1982 . 